Effect of vitamin D supplementation on endothelial function An updated systematic review with meta-analysis and meta-regression

\author{
Pincombe, NL
}

http://hdl.handle.net/10026.1/16430

10.1016/j.numecd.2019.08.005

Nutrition, Metabolism and Cardiovascular Diseases

Elsevier BV

All content in PEARL is protected by copyright law. Author manuscripts are made available in accordance with publisher policies. Please cite only the published version using the details provided on the item record or document. In the absence of an open licence (e.g. Creative Commons), permissions for further reuse of content should be sought from the publisher or author. 


\title{
Effect of vitamin D supplementation on endothelial function - An updated systematic review with meta-analysis and meta-regression
}

\author{
Nick L. Pincombe ${ }^{\mathrm{a}}$, Melissa J. Pearson ${ }^{\mathrm{a}}$, Neil A. Smart ${ }^{\mathrm{a}}$, Nicola King ${ }^{\mathrm{b}}$, Gudrun Dieberg ${ }^{\mathrm{a}, *}$ \\ ${ }^{a}$ School of Science and Technology, University of New England, Armidale, NSW 2351, Australia \\ ${ }^{b}$ School of Biomedical Sciences, Faculty of Medicine and Dentistry, University of Plymouth, Drake's Circus, Plymouth, PL4 8AA, UK
}

Received 17 January 2019; received in revised form 6 August 2019; accepted 6 August 2019

Handling Editor: A. Siani

Available online 19 August 2019

\section{KEYWORDS \\ Vitamin D; \\ Atherosclerosis; Endothelial function; Endothelial dysfunction}

\begin{abstract}
Background and aims: Atherogenesis and endothelial dysfunction contribute to cardiovascular risk and vitamin $\mathrm{D}$ has been implemented in endothelial repair. This systematic review, meta-analysis and meta-regression aims to establish the effect of vitamin D supplementation on endothelial function.

Methods and Results: To conduct the systematic review we searched the Cochrane Library of Controlled Trials, PubMed, ProQuest and EMBASE for randomized controlled trials that investigated the effects of vitamin D supplementation on flow-mediated dilation (FMD\%), pulse wave velocity (PWV), and central augmentation index (AIx). Meta-analysis was based on a random effects model and inverse-variance methods to calculate either mean difference (MD) or standardized mean difference (SMD) as effects sizes. This was followed by meta-regression investigating the effect of baseline vitamin D concentrations, vitamin D dosing and study duration. Risk of bias was assessed using the JADAD scale and funnel plots.

We identified 1056 studies of which 26 studies met inclusion criteria for quantitative analysis. Forty-two percent of the 2808 participants had either deficient or insufficient levels of vitamin D. FMD\% (MD 1.17\% (95\% CI -0.20, 2.54), $p=0.095$ ), PWV (SMD $-0.09 \mathrm{~m} / \mathrm{s}$ (95\% CI -0.24, 0.07), $p=0.275)$ and AIx (SMD 0.05\% (95\% CI -0.1, 0.19), $p=0.52$ ) showed no improvement with vitamin D supplementation. Sub-analysis and meta-regression revealed a tendency for AIx and FMD\% to increase as weekly vitamin doses increased; no other significant relationships were identified.

Conclusions: Vitamin D supplementation showed no improvement in endothelial function. More evidence is required before recommendations for management of endothelial dysfunction can be made.

(c) 2019 The Italian Society of Diabetology, the Italian Society for the Study of Atherosclerosis, the Italian Society of Human Nutrition, and the Department of Clinical Medicine and Surgery, Federico II University. Published by Elsevier B.V. All rights reserved.
\end{abstract}

\footnotetext{
* Corresponding author. Biomedical Science, School of Science and Technology, Faculty of Science, Agriculture, Business and Law, University of New England, NSW 2351, Australia. Fax: +61 267733267.

E-mail address: gdieberg@une.edu.au (G. Dieberg).
}

\section{Introduction}

Cardiovascular disease (CVD) is the leading cause of death globally, with an estimated 17.7 million deaths from the disease worldwide in 2015 [1]. Global CVD burden is estimated to increase to USD 1044 billion by 2030 [2]. Atherosclerosis is indicated as the primary cause of CVD 
and the critical step in CVD commencement and progression with endothelial dysfunction (ED) and associated loss of endothelial integrity presenting as a precursor stage of atherosclerosis [3].

The vascular endothelium can be classified as a complex paracrine and endocrine organ that produces vasodilatory and vasoconstricting factors, most importantly, nitric oxide (NO) [4]. Endothelial dysfunction encompasses dysregulation of mechanistic processes facilitating vasodilatory function of the endothelium, and the actual functionality of the endothelium itself [5]. This broadens the assessments of ED beyond the simple scope of the physical functionality of the endothelial wall and expands the scope to include platelet aggregation and adhesion, vascular inflammation and thrombosis management [5]. Endothelial dysfunction is characterised by a decrease in bioavailable NO leading to a reduction in endothelial vasodilation in response to vasodilatory stimuli [6]. Endothelial dysfunction is the initial, and reversible step in atherogenesis development, and is used as a primary tool for clinical identification of the presence and extent of atherosclerosis by measuring endothelial vasoreactivity $[5,7]$. Measurement of ED is used as a predictor of CVD mortality risk [8]. Endothelial dysfunction is measured through both invasive and non-invasive methods, the most common being central augmentation index percentage (AIx), pulse wave velocity (PWV), and artery flowmediated dilation (FMD\%), the latter considered as the gold standard of ED assessment [5].

Vitamin D is a secosteroid hormone most commonly associated with calcium regulation regarding bone remodelling [9]. Lower serum concentrations of vitamin D have been associated with increased risk of CVD pathogenesis, including increased risk of hypertension, decreased myocyte contractility and increased arterial calcification [10]. Vitamin D receptors are expressed in numerous tissues, and due to their expression in cardiovascular tissues, focus has shifted to vitamin D effects on CVD risk factors, including endothelial function [9]. Speculation exists for the mechanisms for which vitamin D affects endothelial function through NO regulation. One study showed that calcitriol, the hormonally active metabolite of vitamin $\mathrm{D}$, increases monocyte differentiation into myeloid angiogenic cells and augments their angiogenic capacity for endothelial repair, as well as increasing endothelial NO synthase expression, increasing endothelial function [11]. Several RCTs have reported equivocal results regarding the effects of vitamin $\mathrm{D}$ supplementation on arterial stiffness, an important measure of CVD risk, continuing the trend of conjecture regarding RCT results of vitamin D and CVD outcomes [9,10,12-16]. The positive effects of vitamin D on reducing CVD risk factors remain questionable due to RCT results being obtained from populations without hypovitaminosis $\mathrm{D}$. This, and the short duration of the RCTs fails to confirm observational study results which show a positive correlation between vitamin D concentrations and CVD risk factor reduction [13]. Given the expression of vitamin D receptors throughout cardiac tissues, it is entirely conceivable that vitamin D supplementation may improve cardiovascular outcomes, with proposed mechanisms including decreasing the effects of chronic inflammation and decreased vascular smooth muscle cell proliferation [17,18].

This systematic review with meta-analysis and metaregression aims to summarise the currently available evidence from RCTs investigating supplemental vitamin D effects on endothelial function in both healthy and clinical populations. The intent is to draw conclusions as to whether supplemental vitamin D can be recommended for prevention of establishment or progression of ED.

\section{Added value to previous meta-analyses on the same topic}

Previous systematic reviews and meta-analyses [9,12,19-23] have reported on the effects of vitamin D supplementation and markers of endothelial function; however, not all included FMD\%, PWV and AIx as a primary outcome measure and only one provided analyses for all three [23]. In addition, a number of new trials have recently been published and are included in the present systematic review incorporating meta-analysis and metaregression.

\section{Methods}

\section{Search strategy}

A systematic search was conducted to identify potential studies using the following electronic databases: the Cochrane Library of Controlled Trials, PubMed, ProQuest and EMBASE until 31st May 2019. Search criteria included numerous terms, both free text and MeSH regarding atherosclerosis, vitamin D2 and vitamin D3, endothelial function, endothelial dysfunction and terms titling methods for assessing endothelial function. Systematic reviews, meta-analyses, and study bibliographies were reviewed for additional studies. Three reviewers (NP, MP, GD) conducted the search and full article eligibility review.

\section{Study selection}

The following criteria were applied for study identification and selection (1) Randomized, double-blinded, placebo controlled trials; (2) studies conducted in all age groups with no additional exclusion criteria applied for sex, health status or CVD risk factors; (3) vitamin D2 or D3 as primary intervention (dose, administration method or route of vitamin D2 or D3 administration, or inclusion of other secondary interventions were not a basis for study exclusion). Studies using the vitamin D receptor activator Paricalcitol were excluded.

\section{Data extraction and outcome measures}

Data extraction was completed by three reviewers (NP, MP, GD). Primary outcomes measured were limited to endothelial function test measurements conducted via FMD\%, PWV and AIx. Studies reporting FMD values were included 
if the measurement was reported as relative FMD percentage (FMD\%) or as absolute FMD (measured in $\mathrm{cm}$, $\mathrm{mm}$ or $\mu \mathrm{m}$ ) in the brachial artery. Where FMD was reported as both absolute FMD and FMD\%, only data from the FMD\% was extracted for analysis. Studies selected presented numerous PWV and AIx measurements and were included; studies that did not state PWV method were excluded. Units of measure reported for PWV outcomes were converted to $\mathrm{m} / \mathrm{s}$.

\section{Data synthesis}

Individual meta-analyses were completed for continuous data utilizing the change in mean and standard deviation (SD) values for the study populations. Where the change in mean and SD was not reported, the pre-intervention mean was subtracted from the post-intervention mean, and the change SD was calculated utilizing study group participant numbers in conjunction with group $p$-values or $95 \% \mathrm{CI}$. Where exact $p$-values or $95 \% \mathrm{CI}$ were not available the SD of the mean difference was imputed using the formula, $\mathrm{SD}=$ square root $\left[\left(\mathrm{SD}_{\text {pre-treatment }}\right)^{2}+\left(\mathrm{SD}_{\text {post-treat- }}\right.\right.$ ment $\left.)^{2}-\left(2 \mathrm{rSD}_{\text {pre-treatment }} \mathrm{X} \mathrm{SD}_{\text {post-treatment }}\right)\right]$ [24], assuming a correlation coefficient $(r)=0.5$, which is considered a conservative estimate. Where the standard error of the mean (SEM) was given instead of the SD, this value was converted to SD [24]. Data from studies that reported median and interquartile range (IQR 1 st and 3rd quarter) was converted to mean and SD using the method described in Wan et al. [25]. Studies that reported data as median and $50 \%$ IQR had data converted to mean and estimated SD assuming the value given was approximately 1.35 standard deviations. Mean differences (MD) in conjunction with random effects inverse variance were applied to pooled data for FMD\% analysis. Standardized Mean Differences (SMD) were utilized in conjunction with a random effects inverse variance to allow for comparison between differing effects sizes and methods of measurement of the outcome between selected studies, as was the case when conducting analyses for both PWV and AIx pooled data groups. Using current guidelines, SMD values of $0.2,0.5$ and 0.8 signify small, medium and large effects sizes, respectively [26]. Placebo group numbers were evenly divided amongst intervention study groups in selected studies that presented data for more than one intervention group. Forest plots were generated to provide visual representation of vitamin $D$ effects on endothelial function. A 95\% CI was selected to report changes in measured outcomes. Sensitivity analysis was conducted using the leave-one-out method for overall assessment of the intervention effect. Where studies did not report data suitable for pooling, a descriptive analysis was included. Meta-regression analysis was performed (NK) to investigate the heterogeneity of results using baseline vitamin $\mathrm{D}$ concentrations, weekly dose of vitamin D supplement and duration of study as covariates with a $5 \%$ level of significance and 95\% confidence intervals [27]. All analyses were carried out and all figures were produced in Comprehensive Meta-Analysis (CMA) V3 (Biostat Inc., NJ, USA).

\section{Heterogeneity and publication bias}

Heterogeneity between included studies was calculated using CMA V3; the $\mathrm{I}^{2}$ test was utilized for consistency appraisal between studies. Values $<25 \%$ show low risk of heterogeneity, whereas $>75 \%$ shows high risk of heterogeneity. Values that fall between 25 and 75\% show moderate risk of heterogeneity [24]. These values together with assessment of funnel plots and Egger's regression test were used to evaluate overall heterogeneity and assess risk of publication bias [28].

\section{Study quality}

Study quality was assessed using the JADAD scale [29]. The maximum score possible is 5 ; with a score of $\leq 3$ indicating high risk of bias, while a score of $>3$ indicated a low risk of bias. Study quality was performed by two assessors (NP, GD).

\section{Results}

Using allocated search criteria, the search of the four selected databases identified 1056 manuscripts. After removal of duplicates, 59 full-text articles were assessed for eligibility. After screening, a total of 32 full-text papers remained for inclusion in the qualitative analysis, of which 26 were eligible for inclusion in the meta-analysis as per PRISMA flow diagram (Fig. 1).

\section{Study characteristics}

Thirty-two studies were included in the qualitative analysis with 26 suitable for inclusion in the meta-analysis. Reasons for exclusion of the six studies from the metaanalysis are detailed in Supplementary Table 1. The total number of participants analyzed for the specified outcomes from the 26 studies [4,10,14-16,30-50] included in the meta-analysis was 2808 . There was a diversity of participants in the studies, with the inclusion of both healthy and clinical populations. Of the 26 studies, six $[4,14,30,42,45,48]$ provided a total of 327 participants diagnosed with Type 2 Diabetes Mellitus (T2DM) with 55 of these participants identified as being diagnosed with T2DM and non-alcoholic fatty liver disease (NAFLD) [30]. Four studies $[15,38,40,50]$ provided a total of 273 participants diagnosed with various stages of Chronic Kidney Disease (CKD). Remaining studies of clinical populations included participants with increased risk of diabetes, polycystic ovarian syndrome (PCOS), human immunodeficiency virus (HIV), peripheral arterial disease (PAD), chronic fatigue, and CVD. Age group amongst participants ranged from 22.4 to 72.9 (average age $54.6 \pm 14.8$ ). Sex distribution was primarily male. Of the 26 included studies, 8 studies included participants with deficient $(<12.5 \mathrm{ng} / \mathrm{ml}), 11$ studies with inadequate $(<20 \mathrm{ng} / \mathrm{ml})$ and 7 studies with adequate $(\geq 20 \mathrm{ng} / \mathrm{ml})$ baseline vitamin D levels for optimal overall health as per National Institutes of Health recommendations - 58\% of participants were 


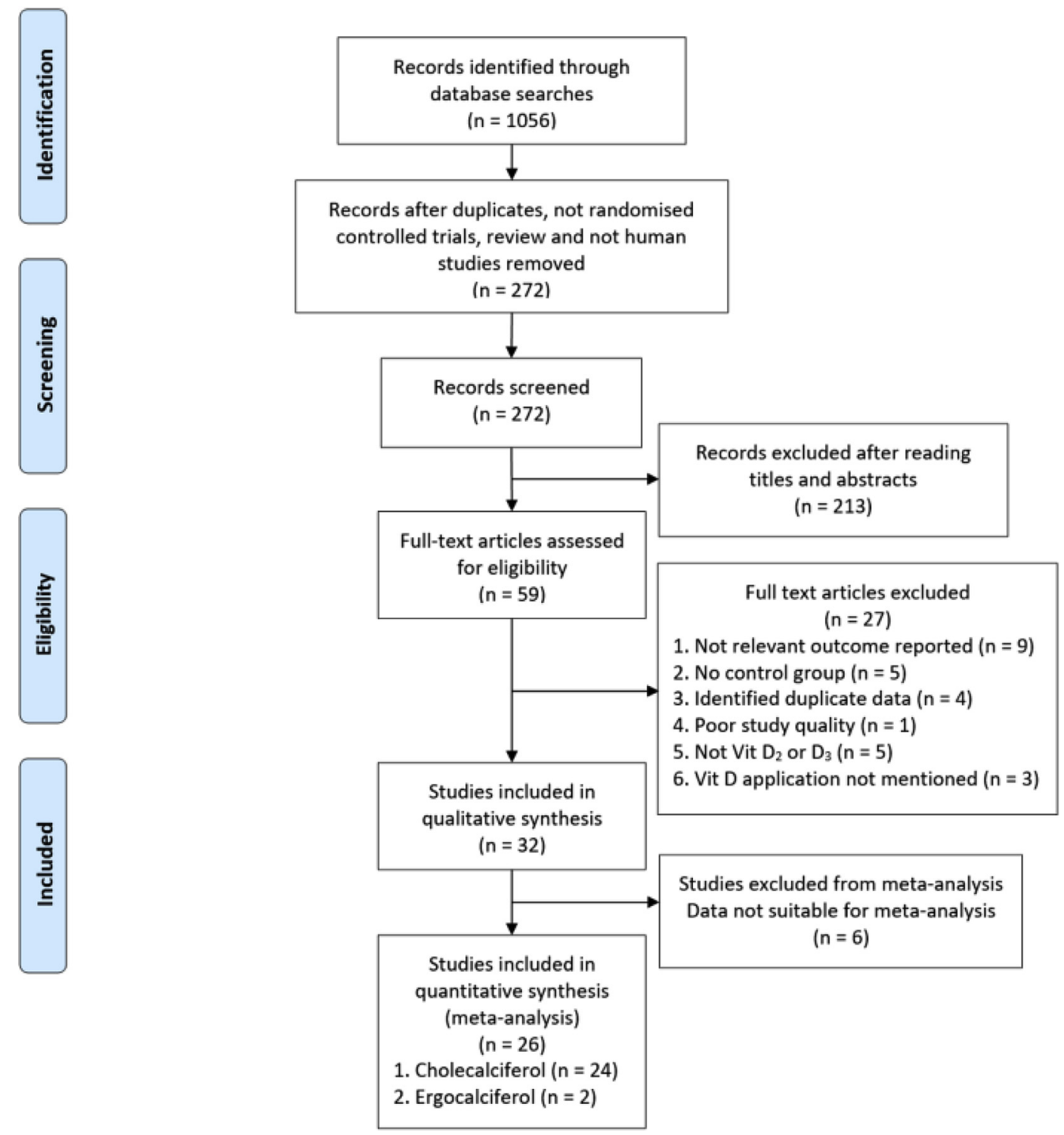

Figure 1 PRISMA flow diagram.

considered to have adequate vitamin D levels. Characteristics of all included studies are detailed in Table 1.

\section{Intervention details}

Intervention duration ranged from four to 57 weeks with most studies using a 16-week intervention period. Vitamin D supplementation dosing ranged from $892 \mathrm{IU} / \mathrm{d}$ to $7142 \mathrm{IU} / \mathrm{d}$, with the higher doses due to singular large dosing either at the start of the study period [44,45,47], or large doses at interval periods longer than daily dosing $[10,15,16,32,33,36-38,43,49,50]$. Accounting for single dose effect over the intervention period most studies used between $2000 \mathrm{IU} / \mathrm{d}$ to $5000 \mathrm{IU} / \mathrm{d}$ and all studies supplemented vitamin D orally. All studies except for two
$[10,45,51]$ used vitamin D3, with one study using both vitamin D2 and D3 for the intervention [32]. In a number of studies, participants were using multiple pharmaceutical treatments in addition to either vitamin D or placebo. One study [33] included dosing of metformin with either 4000 IU of vitamin D plus $1500 \mathrm{mg}$ of metformin, or placebo plus $1500 \mathrm{mg}$ of metformin. One study included use of HIV medications [37] with either vitamin D or placebo. One study included $200 \mathrm{mg} / \mathrm{d}$ of calcium [42] with either vitamin $\mathrm{D}$ or placebo. Dosing intervals ranged from daily dosing to once every three months. To test endothelial function several methods were used across all studies, with the most common being FMD\% [10,15,30,34,35, 37,45,47-49], PWV [15,16,31-34,36,38,40-43,50] and AIx $[4,14,31,33,34,36,38,40,42-44,46,47,49]$. 


\begin{tabular}{|c|c|c|c|c|c|c|c|c|c|}
\hline First Author & $\begin{array}{l}\text { Study } \\
\text { Design }\end{array}$ & Health Status & $\begin{array}{l}\mathrm{n}=\text { analysed } \\
\text { for specified } \\
\text { outcomes }\end{array}$ & $\begin{array}{l}\mathrm{n}=\text { analysed } \\
\text { Vitamin } \mathrm{D} \\
\text { group (VD) }\end{array}$ & Dose & $\begin{array}{l}\mathrm{n}=\text { analysed } \\
\text { placebo } \\
\text { group }(\mathrm{C})\end{array}$ & $\begin{array}{l}\text { Duration } \\
\text { In weeks }\end{array}$ & $\begin{array}{l}\text { Baseline } \\
\text { Vitamin D } \\
\text { Concentration } \\
\mathrm{ng} / \mathrm{ml} \pm \mathrm{SD} \text { or } \mathrm{IQR}\end{array}$ & $\begin{array}{l}\text { Outcome (Vitamin } \\
\text { D versus Placebo) }\end{array}$ \\
\hline $\begin{array}{l}\text { Barchetta } 2016 \\
\quad \text { Italy }\end{array}$ & $\begin{array}{l}\mathrm{R}, \mathrm{DB} \\
\text { placebo }\end{array}$ & T2DM with NAFLD & 55 & 26 & $2000 \mathrm{IU} / \mathrm{d}\left(\mathrm{D}_{3}\right)$ & 29 & 24 & $\begin{array}{l}16.06 \pm 9.56(\mathrm{C}) \\
19.26 \pm 9.48(\mathrm{VD})\end{array}$ & No effect on FMD \\
\hline $\begin{array}{l}\text { Borgi } 2017 \\
\quad \text { United States }\end{array}$ & $\begin{array}{l}\mathrm{R}, \mathrm{DB}, \\
\text { placebo }\end{array}$ & Overweight/obese & 84 & 43 & $50,000 \mathrm{IU} / \mathrm{w}\left(\mathrm{D}_{2}\right)$ & 41 & 8 & 15.4 (IQR 11.4-17.5) & No effect on FMD \\
\hline $\begin{array}{l}\text { Breslavsky } 2013 \\
\text { Israel }\end{array}$ & $\begin{array}{l}\mathrm{R}, \mathrm{DB} \\
\text { placebo }\end{array}$ & T2DM & 32 & 19 & $1000 \mathrm{IU} / \mathrm{d}\left(\mathrm{D}_{3}\right)$ & 13 & 52 & $\begin{array}{l}11.7 \pm 6.5(\mathrm{C}) \\
11.8 \pm 10.9(\mathrm{VD})\end{array}$ & Improved AIx \\
\hline $\begin{array}{l}\text { Bressendorff } \\
2016 \text { Denmark }\end{array}$ & $\begin{array}{l}\mathrm{R}, \mathrm{DB}, \\
\text { placebo }\end{array}$ & Healthy & 40 & 22 & $3000 \mathrm{IU} / \mathrm{d}\left(\mathrm{D}_{3}\right)$ & 18 & 16 & $\begin{array}{l}12.8 \pm 4(\mathrm{C}) \\
12.4 \pm 4.4(\mathrm{VD})\end{array}$ & $\begin{array}{l}\text { No effect on PWV } \\
\text { No effect on AIx }\end{array}$ \\
\hline $\begin{array}{l}\text { Dalan } 2016 \\
\text { Singapore }\end{array}$ & $\begin{array}{l}\text { PG, R, DB, } \\
\text { placebo }\end{array}$ & T2DM & 61 & 31 & $2000-4000 \mathrm{IU} / \mathrm{d}\left(\mathrm{D}_{3}\right)$ & 30 & 16 & $\begin{array}{l}17 \pm 11(\mathrm{C}) \\
18 \pm 7(\mathrm{VD})\end{array}$ & No effect on AIx \\
\hline $\begin{array}{l}\text { Forouhi } 2016 \\
\text { United } \\
\text { Kingdom }\end{array}$ & $\begin{array}{l}\text { R, DB, } \\
\text { placebo }\end{array}$ & $\begin{array}{l}\text { Increased risk } \\
\text { T2DM }\end{array}$ & 160 & $\begin{array}{l}55 \\
53\end{array}$ & $\begin{array}{l}100,000 \mathrm{IU} / \mathrm{m}\left(D_{2}\right) \\
100,000 \mathrm{IU} / \mathrm{m}\left(D_{3}\right)\end{array}$ & 52 & 16 & $\begin{array}{l}18.32 \pm 10.52(\mathrm{C}) \\
19.44 \pm 9.88(\mathrm{VD} 2) \\
18.32 \pm 9.04(\mathrm{VD} 3)\end{array}$ & Improved PWV \\
\hline $\begin{array}{l}\text { Garg } 2015 \\
\quad \text { India }\end{array}$ & $\begin{array}{l}\mathrm{P}, \mathrm{R}, \mathrm{DB} \\
\text { placebo }\end{array}$ & PCOS & 32 & 15 & $120,000 \mathrm{IU} / \mathrm{m}\left(\mathrm{D}_{3}\right)$ & 17 & 24 & $\begin{array}{l}6.8 \pm 2.46(\mathrm{C}) \\
7.7 \pm 6.05(\mathrm{VD})\end{array}$ & $\begin{array}{l}\text { No effect on PWV } \\
\text { No effect on AIx }\end{array}$ \\
\hline $\begin{array}{l}\text { Gepner } 2012 \\
\quad \text { United States }\end{array}$ & $\begin{array}{l}\mathrm{P}, \mathrm{R}, \mathrm{DB} \\
\text { placebo }\end{array}$ & $\begin{array}{l}\text { Healthy, } \\
\text { post-menopausal }\end{array}$ & $\begin{array}{l}109 \text { (FMD) } \\
75 \text { (PWV \& Alx) }\end{array}$ & $\begin{array}{l}55 \text { (FMD) } \\
38 \text { (PWV \& Alx) }\end{array}$ & $2500 \mathrm{IU} / \mathrm{d}\left(\mathrm{D}_{3}\right)$ & $\begin{array}{l}54 \text { (FMD) } \\
37 \text { (PWV \& Alx) }\end{array}$ & 16 & $\begin{array}{l}32.3 \pm 10.5(\mathrm{C}) \\
30.3 \pm 10.7(\mathrm{VD})\end{array}$ & $\begin{array}{l}\text { No effect on FMD } \\
\text { No effect on PWV } \\
\text { No effect on AIX }\end{array}$ \\
\hline $\begin{array}{l}\text { Harris } 2011 \\
\text { United States }\end{array}$ & $\begin{array}{l}\mathrm{P}, \mathrm{R}, \mathrm{DB}, \\
\text { placebo }\end{array}$ & Overweight & 45 & 22 & $60,000 \mathrm{IU} / \mathrm{m}\left(\mathrm{D}_{3}\right)$ & 23 & 16 & $\begin{array}{l}15.28 \pm 1.2(\mathrm{C}) \\
13.72 \pm 0.88(\mathrm{VD})\end{array}$ & Improved FMD \\
\hline $\begin{array}{l}\text { Kumar } 2017 \\
\quad \text { India }\end{array}$ & $\begin{array}{l}\mathrm{R}, \mathrm{DB} \\
\text { placebo }\end{array}$ & CKD & 117 & 58 & $\begin{array}{l}300,000 \text { IU at baseline } \\
\text { and } 8 \mathrm{w}\left(\mathrm{D}_{3}\right)\end{array}$ & 59 & 16 & $\begin{array}{l}13.21 \pm 4.78(\mathrm{C}) \\
13.4 \pm 4.42(\mathrm{VD})\end{array}$ & $\begin{array}{l}\text { Improved FMD } \\
\text { Improved PWV }\end{array}$ \\
\hline $\begin{array}{l}\text { Larsen } 2012 \\
\text { Denmark }\end{array}$ & $\begin{array}{l}\mathrm{R}, \mathrm{DB} \\
\text { placebo }\end{array}$ & Hypertension & $\begin{array}{l}\text { 111(PWV) } \\
107 \text { (Alx) }\end{array}$ & $\begin{array}{l}54 \\
52\end{array}$ & $3000 \mathrm{IU} / \mathrm{d}\left(\mathrm{D}_{3}\right)$ & $\begin{array}{l}57 \\
55\end{array}$ & 20 & $\begin{array}{l}23 \pm 12(\mathrm{C}) \\
23 \pm 9(\mathrm{VD})\end{array}$ & $\begin{array}{l}\text { No effect on PWV } \\
\text { No effect on AIx }\end{array}$ \\
\hline $\begin{array}{l}\text { Levin } 2017 \\
\text { Canada }\end{array}$ & $\begin{array}{l}\mathrm{R}, \mathrm{DB}, \\
\text { placebo }\end{array}$ & CKD & 87 & 57 & $15,000 \mathrm{IU} / \mathrm{w}$ (D3) & 30 & 21 & $\begin{array}{l}29.4 \pm 12.7(\mathrm{C}) \\
25.9 \pm 10(\mathrm{VD})\end{array}$ & Improved PWV \\
\hline $\begin{array}{l}\text { Longnecker } 2012 \\
\text { United States }\end{array}$ & $\begin{array}{l}\mathrm{P}, \mathrm{R}, \mathrm{DB} \\
\text { placebo }\end{array}$ & HIV & 45 & 30 & $4000 \mathrm{IU} / \mathrm{d}\left(\mathrm{D}_{3}\right)$ & 15 & 12 & $\begin{array}{l}6.2(\text { IQR 3.7-9.8) (C) } \\
9 \text { (IQR 7.1-13.1) (VD) }\end{array}$ & No effect on FMD \\
\hline $\begin{array}{l}\text { Marckmann } \\
2012 \text { Denmark }\end{array}$ & $\begin{array}{l}\mathrm{PG}, \mathrm{R}, \mathrm{DB}, \\
\text { placebo }\end{array}$ & CKD & $\begin{array}{l}28 \text { (PWV) } \\
23 \text { (Alx) }\end{array}$ & $\begin{array}{l}12(\mathrm{PWV}) \\
9 \text { (Alx) }\end{array}$ & $40,000 \mathrm{IU} / \mathrm{w}\left(\mathrm{D}_{3}\right)$ & $\begin{array}{l}\text { 16(PWV) } \\
14(\mathrm{Alx})\end{array}$ & 8 & $\begin{array}{l}13.2 \text { (IQR 9.4-17.1)(C) } \\
9.5(\mathrm{IQR} 6.9-16.6)(\mathrm{VD})\end{array}$ & $\begin{array}{l}\text { No effect on PWV } \\
\text { No effect on AIx }\end{array}$ \\
\hline $\begin{array}{l}\text { Martins } 2014 \\
\text { United States }\end{array}$ & $\begin{array}{l}\mathrm{R}, \mathrm{DB} \\
\text { placebo }\end{array}$ & Overweight/obese & 130 & 65 & $100,000 \mathrm{IU} / \mathrm{m}\left(\mathrm{D}_{3}\right)$ & 65 & 12 & $\begin{array}{l}6.6 \pm 2(\mathrm{C}) \\
6.8 \pm 2.08(\mathrm{VD})\end{array}$ & No effect AIx \\
\hline $\begin{array}{l}\text { Mose } 2014 \\
\text { Denmark }\end{array}$ & $\begin{array}{l}\mathrm{R}, \mathrm{DB}, \\
\text { placebo }\end{array}$ & $\begin{array}{l}\text { CKD stage } 5- \\
\text { chronic dialysis }\end{array}$ & $\begin{array}{l}41 \text { (PWV) } \\
40 \text { (AIx) }\end{array}$ & $\begin{array}{l}22 \\
18\end{array}$ & $3000 \mathrm{IU} / \mathrm{d}\left(\mathrm{D}_{3}\right)$ & $\begin{array}{l}19 \\
22\end{array}$ & 24 & $\begin{array}{l}11.2 \text { (IQR 8-27.6) (C); } \\
11.2 \text { (IQR 8-19.2) (VD) }\end{array}$ & $\begin{array}{l}\text { No effect on PWV } \\
\text { Improved AIx }\end{array}$ \\
\hline $\begin{array}{r}\text { Pilz } 2015 \\
\text { Austria }\end{array}$ & $\begin{array}{l}\mathrm{R}, \mathrm{DB} \\
\text { placebo }\end{array}$ & Hypertension & 153 & 81 & $2800 \mathrm{IU} / \mathrm{d}\left(\mathrm{D}_{3}\right)$ & 72 & 8 & $\begin{array}{l}20.4 \pm 5.7(C) \\
22.0 \pm 5.5(V D)\end{array}$ & No effect on PWV \\
\hline $\begin{array}{l}\text { Raed } 2017 \\
\quad \text { United States }\end{array}$ & $\begin{array}{l}\mathrm{R}, \mathrm{DB}, \\
\text { placebo }\end{array}$ & Overweight & 70 & $\begin{array}{l}17 \\
18 \\
18\end{array}$ & $\begin{array}{l}18,000 \mathrm{IU} / \mathrm{m}\left(\mathrm{D}_{3}\right) \\
60,000 \mathrm{IU} / \mathrm{m}\left(\mathrm{D}_{3}\right) \\
120,000 \mathrm{IU} / \mathrm{m}\left(\mathrm{D}_{3}\right)\end{array}$ & 17 & 16 & $\begin{array}{l}15.9 \pm 3.9(\mathrm{C}) \\
14 \pm 3.9(\mathrm{VD}-18,000) \\
15.9 \pm 3.7(\mathrm{VD}-60,000) \\
13.3 \pm 4.4(\mathrm{VD}-120,000)\end{array}$ & Improved PWV \\
\hline $\begin{array}{l}\text { Ryu } 2014 \\
\text { Korea }\end{array}$ & $\begin{array}{l}\mathrm{P}, \mathrm{R}, \mathrm{DB}, \\
\text { placebo }\end{array}$ & T2DM & 45 & 24 & $2000 \mathrm{IU} / \mathrm{d}\left(\mathrm{D}_{3}\right)$ & 21 & 24 & $\begin{array}{l}10.7 \pm 2.6(\mathrm{C}) \\
12.3 \pm 3.0(\mathrm{VD})\end{array}$ & $\begin{array}{l}\text { No effect on PWV } \\
\text { No effect on AIx } \\
\text { tinued on next page) }\end{array}$ \\
\hline
\end{tabular}




\begin{tabular}{|c|c|c|c|c|c|c|c|c|c|}
\hline First Author & $\begin{array}{l}\text { Study } \\
\text { Design }\end{array}$ & Health Status & $\begin{array}{l}\mathrm{n}=\text { analysed } \\
\text { for specified } \\
\text { outcomes }\end{array}$ & $\begin{array}{l}\mathrm{n}=\text { analysed } \\
\text { Vitamin } \mathrm{D} \\
\text { group }(\mathrm{VD})\end{array}$ & Dose & $\begin{array}{l}\mathrm{n}=\text { analysed } \\
\text { placebo } \\
\text { group }(\mathrm{C})\end{array}$ & $\begin{array}{l}\text { Duration } \\
\text { In weeks }\end{array}$ & $\begin{array}{l}\text { Baseline } \\
\text { Vitamin D } \\
\text { Concentration } \\
\mathrm{ng} / \mathrm{ml} \pm \mathrm{SD} \text { or IQR }\end{array}$ & $\begin{array}{l}\text { Outcome (Vitamin } \\
\text { D versus Placebo) }\end{array}$ \\
\hline $\begin{array}{l}\text { Sluyter } 2017 \\
\quad \text { New Zealand }\end{array}$ & $\begin{array}{l}\mathrm{R}, \mathrm{DB}, \\
\text { placebo }\end{array}$ & $\begin{array}{l}\text { Aged } 50-84 \text { yrs } \\
(>60 \% \text { HT })\end{array}$ & 517 & 256 & $\begin{array}{l}200,000\left(D_{3}\right) \text { initially, } \\
\text { then } 100,000 \mathrm{IU} / \mathrm{m}\end{array}$ & 261 & 57 & $\begin{array}{l}25.24 \pm 9.84(C) \\
24.84 \pm 9.88(V D)\end{array}$ & $\begin{array}{l}\text { No effect on PWV } \\
\text { No effect on Alx } \\
\text { (improved in Vit D } \\
\text { deficient subgroup) }\end{array}$ \\
\hline $\begin{array}{r}\text { Stricker } 2012 \\
\text { Switzerland }\end{array}$ & $\begin{array}{l}\text { Pilot, R, } \\
\text { DB, } \\
\text { placebo }\end{array}$ & PAD & 62 & 31 & $\begin{array}{l}100,000 \mathrm{IU} \text { - single } \\
\text { dose }\left(D_{3}\right)\end{array}$ & 31 & 4 & $\begin{array}{l}17 \pm 5.5(\mathrm{C}) \\
16.3 \pm 6.7(\mathrm{VD})\end{array}$ & No effect on Alx \\
\hline $\begin{array}{l}\text { Sugden } 2008 \\
\text { United } \\
\text { Kingdom }\end{array}$ & $\begin{array}{l}\text { P, PG, DB, } \\
\text { R placebo }\end{array}$ & T2DM & 34 & 17 & $\begin{array}{l}100,000 \mathrm{IU} \text { - single } \\
\text { dose }\left(D_{2}\right)\end{array}$ & 17 & 8 & $\begin{array}{l}14.56 \pm 3.4(\mathrm{C}) \\
16.08 \pm 4.12(\mathrm{VD})\end{array}$ & Improved FMD \\
\hline $\begin{array}{l}\text { Tomson } 2017 \\
\text { United } \\
\text { Kingdom }\end{array}$ & $\begin{array}{l}\text { PG, R, DB, } \\
\text { placebo }\end{array}$ & Aged $\geq 65$ yrs & 305 & $\begin{array}{l}102 \\
102\end{array}$ & $\begin{array}{l}2000 \mathrm{IU} / \mathrm{d}\left(D_{3}\right) \\
4000 \mathrm{IU} / \mathrm{d}\left(D_{3}\right)\end{array}$ & 101 & 52 & 20 - all groups & $\begin{array}{l}\text { No effect on PWV } \\
\text { No effect on AIx }\end{array}$ \\
\hline $\begin{array}{l}\text { Witham 2013b } \\
\text { United } \\
\text { Kingdom - } \\
\text { South Asian }\end{array}$ & $\begin{array}{l}\text { PG, R, DB, } \\
\text { placebo }\end{array}$ & Healthy & 50 & 25 & $\begin{array}{l}100,000 \mathrm{IU} \text { - single } \\
\text { dose }\left(D_{3}\right)\end{array}$ & 25 & 8 & $\begin{array}{l}10.8 \pm 6(\mathrm{C}) \\
10.8 \pm 5.2(\mathrm{VD})\end{array}$ & $\begin{array}{l}\text { No effect on FMD } \\
\text { No effect on PWV } \\
\text { No effect on AIx }\end{array}$ \\
\hline $\begin{array}{l}\text { Witham } 2015 \\
\text { United } \\
\text { Kingdom }\end{array}$ & $\begin{array}{l}\text { PG, R, DB, } \\
\text { placebo }\end{array}$ & Chronic Fatigue & 50 & 25 & $\begin{array}{l}100,000 \mathrm{IU} / 2 \\
\text { months }\left(\mathrm{D}_{3}\right)\end{array}$ & 25 & 24 & $\begin{array}{l}19.2 \pm 8(C) \\
17.6 \pm 6(V D)\end{array}$ & $\begin{array}{l}\text { No effect on FMD } \\
\text { No effect on PWV }\end{array}$ \\
\hline $\begin{array}{l}\text { Yiu } 2013 \\
\text { China }\end{array}$ & $\begin{array}{l}\mathrm{P}, \mathrm{R}, \mathrm{DB} \\
\text { placebo }\end{array}$ & T2DM & 100 & 50 & $5000 \mathrm{IU} / \mathrm{d}\left(\mathrm{D}_{3}\right)$ & 50 & 12 & $\begin{array}{l}21.9 \pm 4.1(\mathrm{C}) \\
21.1 \pm 4.4(\mathrm{VD})\end{array}$ & $\begin{array}{l}\text { No effect on FMD } \\
\text { No effect on PWV }\end{array}$ \\
\hline
\end{tabular}




\section{Outcome measures}

\section{Flow-mediated dilation}

Ten studies assessed FMD\% as either a primary or secondary outcome and included a total of 689 participants $[10,15,30,34,35,37,45,47-49]$. Vitamin D supplementation did not improve FMD\% with MD 1.17\% (95\% CI -0.20, 2.54 ), $p=0.095$ (Fig. 2). Three additional studies [52-54] reported on FMD\%, however, data did not allow for pooling. All three of these studies reported no change in FMD\% with vitamin D supplementation. Sensitivity analysis for FMD\% indicated that the studies of Kumar (2017) [15] and Harris (2011) [35] impacted the size and significance of the result. Upon removal of these studies, MD was reduced to $0.67 \%$ (95\% CI $-0.55,1.88), p=0.28$ and $0.92 \%$ (95\% CI $-0.66,2.49), p=0.25$, respectively (Supplementary Table 2).

\section{Pulse wave velocity}

Seventeen studies assessed PWV as either a primary or secondary outcome $[15,16,31-34,36,38,40-43,50]$ and included a total of 1981 participants. Vitamin D supplementation did not improve PWV with SMD $-0.09 \mathrm{~m} / \mathrm{s}$ (95\% CI -0.24, 0.07), $p=0.275$ (Fig. 3). An additional four studies $[51,52,55,56]$ reported on PWV (data did not allow for pooling) and showed no change in PWV with Vitamin D supplementation. Leave-one-out sensitivity analysis for PWV did not indicate that any study overly impacted the results (Supplementary Table 3).

\section{Augmentation index percentage}

Fifteen studies assessed AIx as either a primary or secondary outcome AIx [4,14,31,33,34,36,38,40,42-44,46,47,49] and included a total of 1569 participants. Vitamin D supplementation did not improve AIx with SMD 0.05\% (95\% CI -0.1, 0.19 ), $p=0.52$ (Fig. 4). An additional study [55] reported on AIx (data did not allow for pooling) and reported no change in AIx with vitamin D supplementation. Leave-one-out sensitivity analysis for AIx did not indicate that any study overly impacted the results (Supplementary Table 4).

\section{Sub-analyses of vitamin D supplementation effect on FMD\%, PWV and AIx}

Sub-analyses were conducted for FMD\%, PWV and AIx on the effect of baseline vitamin D levels (deficient, inadequate or adequate), vitamin D supplementation (daily, weekly, monthly or 2-monthly), the amount of vitamin D administered ( $<20,000 \mathrm{IU} /$ week or $>20,000 \mathrm{IU} /$ week), and in populations with T2DM and CKD (Supplementary Figures 1 to 12).

Baseline vitamin D levels seemed to improve FMD\% in those participants with inadequate vitamin D levels with MD 1.8\% (95\% CI -0.06, 3.68), $p=0.057$. In participants with deficient or inadequate baseline vitamin D levels, PWV showed some improvement in vitamin $\mathrm{D}$ deficient participants with SMD $0.29 \mathrm{~m} / \mathrm{s}$ (95\% CI -0.01, 0.6), $p=0.06$ and was significantly decreased in participants with inadequate vitamin $\mathrm{D}$ levels, favouring the placebo group, with $-0.38 \mathrm{~m} / \mathrm{s}$ (95\% CI $-0.09,-2.59), p=0.01$, respectively. AIx was not affected by baseline vitamin $\mathrm{D}$ levels (Supplementary Figures 1-3).

The timing of vitamin D supplementation did not significantly improve FMD\%, PWV or AIx when supplemented daily, weekly, monthly or 2-monthly (Supplementary Figures 4-6).

Vitamin D dosage of $<20,000 \mathrm{IU} /$ week vs. $>20,000 \mathrm{IU} /$ week did not significantly affect FMD\% or PWV; however, AIx showed a significant improvement when doses of $>20,000$ IU/week were administered with SMD 0.13\% (95\% CI 0.015, 0.25), $p=0.03$ (Supplementary Figures 7-9).

Type 2 Diabetes - Pooled data for FMD\%, PWV and Alx on Vitamin D supplementation in populations with T2DM did not demonstrate a significant improvement in any measure (Supplementary Figures 10 and 12). One

Flow Mediated Dilation

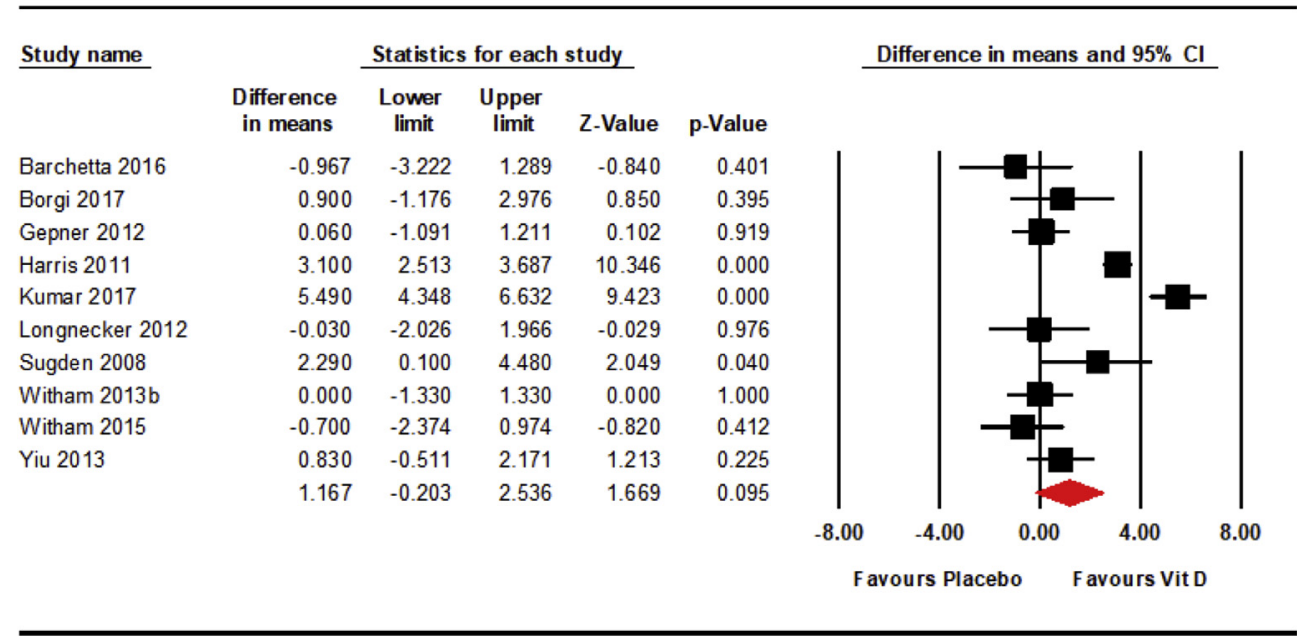

Figure 2 FMD\%: Vitamin D supplementation vs placebo. 


\section{Pulse Wave Velocity}

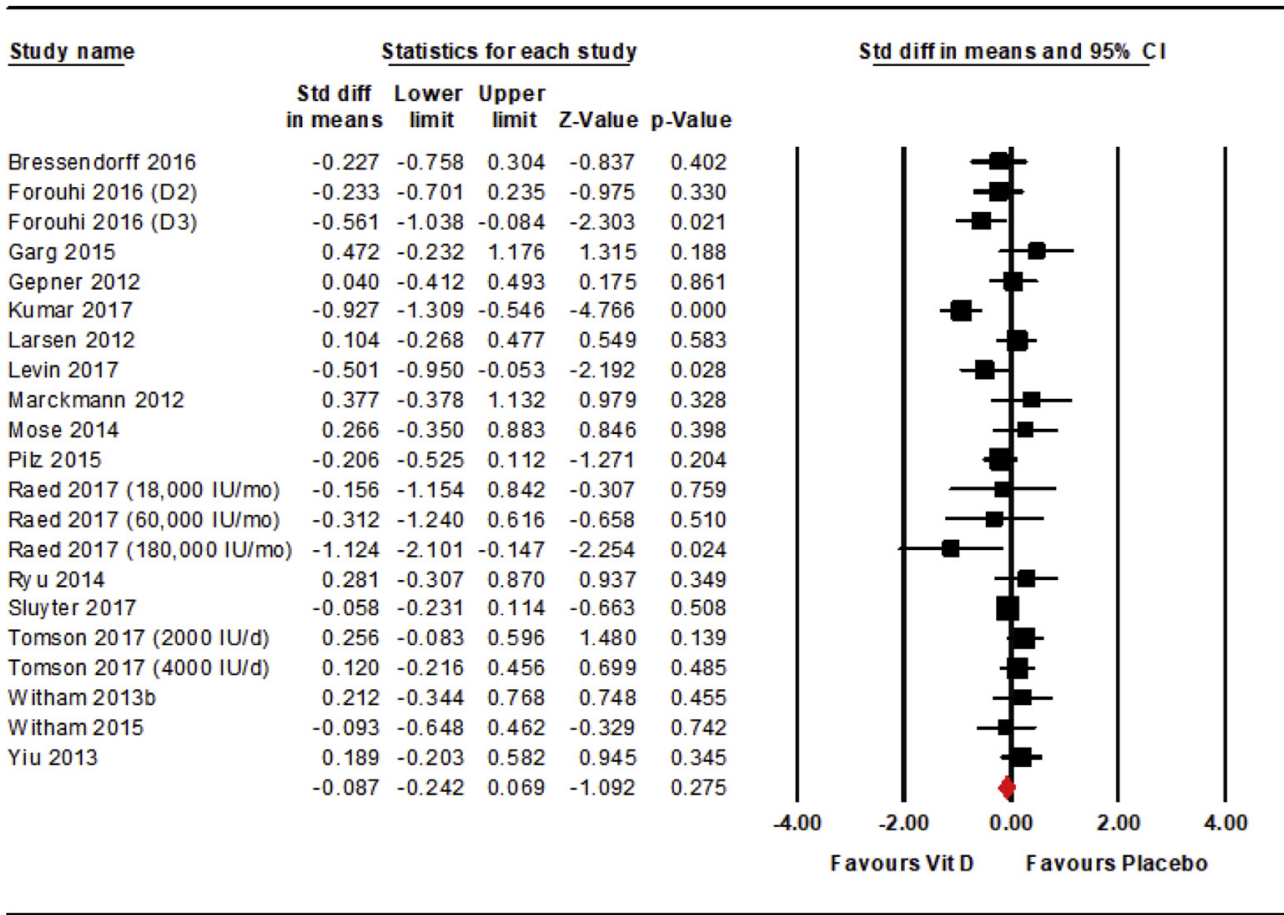

Figure 3 PWV: Vitamin D supplementation vs placebo.

\section{Augmentation Index}

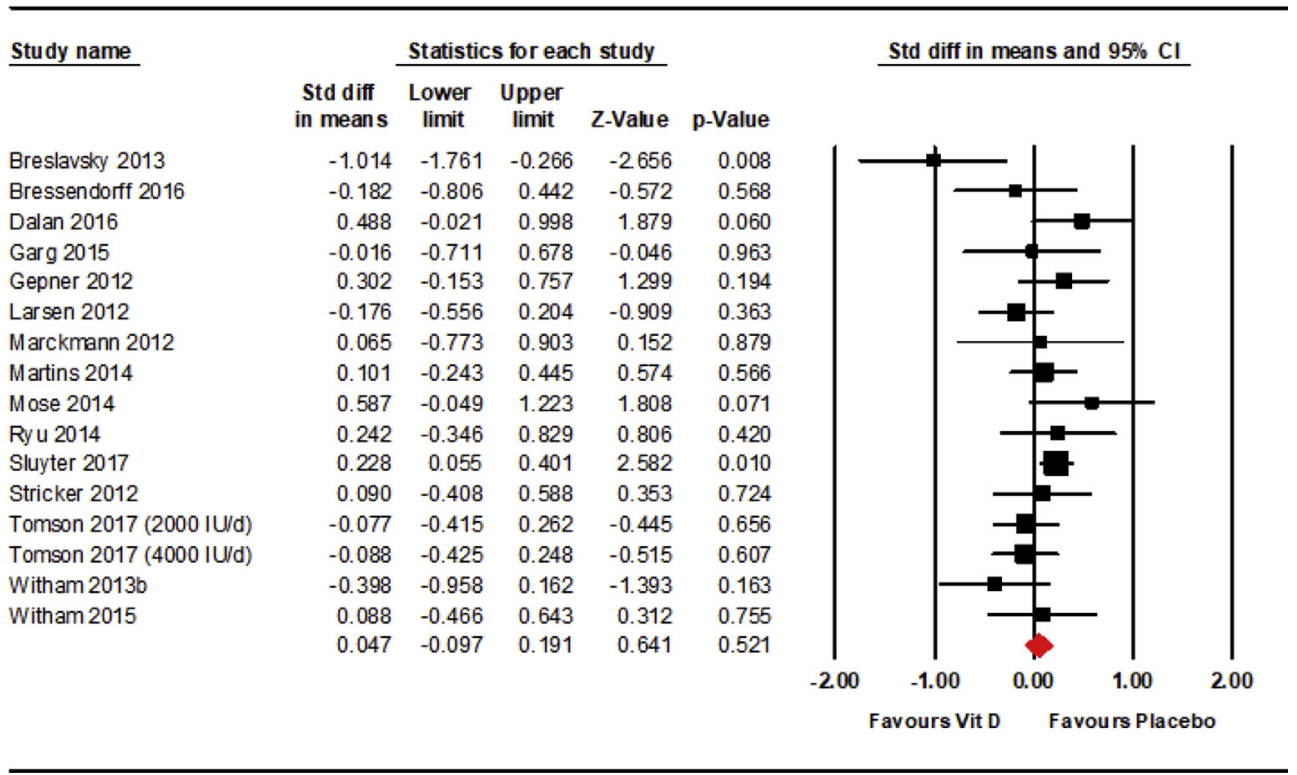

Figure 4 AIx: Vitamin D supplementation vs placebo.

additional study in a T2DM population [54], not included in the pooled analysis also failed to find any significant improvement.

Chronic Kidney Disease - Only one study [15] included in the review reported FMD\% in a population with CKD; vitamin D supplementation improved FMD\% with MD
5.49\% (95\% CI 4.35, 6.63), $p=0.00$. Subgroup analyses for PWV (4 RCTs) and Alx (2 RCTs) failed to indicate any significant improvement (Supplementary Figures 10 and 12). Two additional studies [51,56] in CKD patients, not included in the pooled analysis, also failed to demonstrate any significant improvements. 


\section{Meta-regression for covariates}

The covariates baseline vitamin D concentration, weekly dose of vitamin D supplement and study duration were investigated for FMD\%, PWV and AIx. There was a tendency for the mean difference of FMD\% to increase as weekly dose of vitamin D supplementation increased $(p=0.05)$. No other significant relationships were found (Supplementary Figures 13-21).

\section{Study quality assessment}

The median JADAD score was 5 out of a maximum score of 5 (Supplementary Table 5). Most studies scored 5; however, a few studies lost points due to either not describing the method of blinding or randomization.

\section{Heterogeneity and publication bias}

Most analyses demonstrated moderate to high heterogeneity with $\mathrm{I}^{2}=90.38 \%$ for FMD $\% \mathrm{I}^{2}=58.23 \%$ for PWV and $\mathrm{I}^{2}=38.07 \%$ for AIx. Egger funnel plots showed little evidence of publication bias with an intercept of -4.05 (95\% $\mathrm{CI}-8.76,0.67), p=0.083$ for FMD\%, -0.16 (95\% CI -1.87 , 1.55), $p=0.85$ for PWV and -0.93 (95\% CI $-2.47,0.61)$, $p=0.217$ for AIx (Supplementary Figures 22-24).

\section{Discussion}

This systematic review with meta-analysis and metaregression found little evidence to support the use of vitamin D supplementation to improve endothelial function. Pooled data failed to demonstrate any statistically significant improvement in FMD\%, PWV and AIx, all of which are considered markers of endothelial function. These results are consistent with previous analyses on vitamin D supplementation and endothelial function $[9,12,21,23]$ and another analysis that has identified a lack of positive effects for treatment of hypertension with vitamin D [57]. Our results and those of these previous analyses contrast with a recent analysis [19], which demonstrated a significant improvement in FMD\%. This analysis differed from ours by conducting the assessment of vitamin D supplementation on FMD\% with studies that did not meet our inclusion criteria and included studies that conducted assessment of supplemental vitamin D and vitamin D analogues [58]. Our analysis included the study by Sluyter (2017) [43] which recruited the largest number of participants to date investigating the effects of vitamin $\mathrm{D}$ on central and brachial blood pressure parameters and failed to find any significant effect of vitamin D supplementation on PWV and AIx. However, a subgroup analysis in participants with vitamin D deficiency indicated significant improvements in PWV and Alx [43].

Results amongst pooled study data are conflicting and possibly due to heterogeneity of vitamin D dosing, dosing schedules, baseline concentrations, duration of intervention, study power $[44,59,60]$ and possible confounding factors which included sunlight exposure, diet, medications and unverified additional supplementation [16,42].
Choice of vitamin D type may also affect RCT results, as new evidence indicates that vitamin D3 is more biologically active than vitamin D2 [61,62]. However, only three out of 26 studies $[10,32,45]$ included in this meta-analysis used vitamin D2 supplementation. Several studies included supplemental or pharmacological interventions as a part of the selected population group, including calcium [42], HIV medications [37] and metformin [33]. Vitamin D receptor genetic variation [63], body mass index and disease-related biases for vitamin D metabolism may have also influenced results that vitamin D supplementation may have a positive effect on populations with T2DM $[14,45]$ and CKD $[15,40,50,64]$. This was not confirmed by recent systematic reviews [22,65]. One study [66] suggest that higher risk of CVD due to hypovitaminosis may be due to a phenotypical trait over one or several specific CVD risk factors, with additional sex-related differences such as hormonal factors, as indicated with women appearing to have an increased risk of a number of CVD risk factors in comparison to men. Standardization of populations for comparison whilst accounting for external factors including environmental, smoking and dietary intake variation increase the challenge for identification of actual long-term effects of vitamin D supplementation.

Individually, only eight trials included in this systematic review reported a significant improvement in FMD\%, PWV or AIx [14-16,32,35,40,45,50], when the intervention groups were compared to the placebo groups. Two of the studies [16,35] were conducted in populations of overweight African-Americans, one of these studies only included participants who were vitamin D deficient [16]. Two of the studies were conducted in populations with T2DM or at risk of T2DM [14,45], one [32] in a population of participants at an increased risk of T2DM. Three studies were conducted in CKD participants $[15,40,50]$.

Our sub-analyses investigated the effects of vitamin D supplementation considering participants' baseline vitamin D levels, dosing schedules, dose of vitamin D administered, and chronic diseases. Participants with deficient or inadequate baseline vitamin D levels tended to show some improvements in PWV and FMD\%, respectively; conflictingly, PWV was significantly decreased in those with inadequate levels of vitamin $\mathrm{D}$ at baseline. Dosing schedules did not improve FMD\%, PWV and AIx; however, higher doses of vitamin D significantly improved the AIx especially in participants with deficient or inadequate baseline vitamin D levels. Sub-analysis of T2DM and CKD participants showed no improvements in endothelial function with only one study of CKD participants [15] finding a significant increase in FMD\%. Populations with CKD are commonly vitamin D deficient [51,67] somewhat due to a reduction in vitamin $D$ receptors and receptor resistance [68-71], impaired vitamin D tubular resorption [72] and reduced hepatic and renal synthesis of 1,25dihydroxycholecalciferol due to decreased hepatic CYP450 enzyme expression and reduced renal $1 \alpha$-hydroxylase [73]. The effectiveness of higher doses of vitamin D supplementation to improve FMD\% may be due to a reduction in renal hyperparathyroidism through vitamin $\mathrm{D}$ 
induced suppression of elevated intact parathyroid hormone $[15,74]$ by increasing catabolism of vitamin $D$ to 1,25 hydroxyvitamin D [75]. Additionally, vitamin D supplementation could increase control of hemodynamic factors through reduction of renin-angiotensin-aldosterone system activity reducing the effects of hypertension on CKD [76-78]. Vitamin D supplementation may improve cardiovascular risk factors in non-diabetic patients in early stages of CKD.

Our meta-regression analyses found no significant relationships for the covariates baseline vitamin D levels, weekly dose of vitamin D or study duration.

Further research is still required to understand the mechanistic effects of vitamin D and other endogenous compounds for prevention of atherosclerosis. Future research in populations that commonly use several supplements, including vitamin D and calcium, may help to determine if combination supplementation can improve endothelial function and reduce the risk of atherosclerosis, such as in post-menopausal females supplementing with calcium to decrease osteoporosis risk. Additionally, analysis of whether nutritional or supplemental sources decrease risk of atherosclerosis would be of benefit.

There are a number of strengths to this paper. No study intervention dose fell below minimum Australian recommended dosing requirements for study participants, being $200 \mathrm{IU} /$ day for populations aged $19-50$ years old, $400 \mathrm{IU} /$ day for populations aged 50-70 years old, and $600 \mathrm{IU} /$ day for populations aged 70 years or older [79]. The meta-analysis utilized data only provided by trials that were designed as placebo-controlled, randomized, double-blind trials. Stringent selection criteria focused specifically on vitamin D2 and $\mathrm{D} 3$, excluding vitamin $\mathrm{D}$ analogues like the commonly prescribed vitamin $D$ receptor activiator Paricalcitol for CKD. The primary rationale for this was to investigate recommendations for over-the-counter options for the consumer to assist with improving endothelial function.

There were several limitations that need to be highlighted for this systematic review and the meta-analysis and meta-regression. The shortest intervention time was four weeks [44] which may be less than the estimated required time period for vitamin $\mathrm{D}$ repletion to occur due to the accepted half-life of vitamin D being eight weeks, and possible variations between half-lives of vitamin D2 and D3 [63]. Most studies used a 16-week intervention period which could be argued as still being too short to observe vitamin $\mathrm{D}$ facilitated changes on surrogate markers of arterial stiffness. Seven studies ran over 20-24 weeks, two studies had a study duration of 1 year, and only one study was conducted for longer than 12 months [43]. Our meta-regression analyses did not confirm any improvements in endothelial function with increased study duration. FMD\%, PWV or AIx were not the primary outcome measures in some studies, hence statistical power may have affected the results of these studies. Only studies that measured FMD\%, PWV and AIx were included; however, several other indices are markers of endothelial function and hence the results need to be interpreted with this context.
In conclusion, this meta-analysis and meta-regression suggests that vitamin D supplementation has no significant effect for improving endothelial dysfunction, although there is a tendency to improve FMD\%. The current evidence is conclusive enough to suggest that supplementation or pharmacological prescription of vitamin $\mathrm{D}$ is not warranted for improving endothelial dysfunction. It is still unclear whether vitamin D supplementation is required for those population groups that require repletion. There is some evidence that vitamin D supplementation may be beneficial for populations with CKD, or overweight populations.

\section{Conflicts of interest}

All authors declare to having no actual or potential conflict of interest including any financial, personal or other relationships with other people or organizations within three years of beginning the submitted work that could inappropriately, or be perceived to influence, their work.

\section{Appendix A. Supplementary data}

Supplementary data to this article can be found online at https://doi.org/10.1016/j.numecd.2019.08.005.

\section{References}

[1] Organization WH. Cardiovascular disease (CVDs). 2017.

[2] Program CA. The cost of CVD. 2018.

[3] Higashi Y, Noma K, Yoshizumi M, Kihara Y. Endothelial function and oxidative stress in cardiovascular diseases. Circ J 2009;73(3):411-8.

[4] Dalan R, Liew H, Assam PN, Chan ES, Siddiqui FJ, Tan AW, et al. A randomised controlled trial evaluating the impact of targeted vitamin D supplementation on endothelial function in type 2 diabetes mellitus: the DIMENSION trial. Diabetes Vasc Dis Res 2016; 13(3):192-200.

[5] Barac A, Campia U, Panza JA. Methods for evaluating endothelial function in humans. Hypertension 2007;49(4):748-60.

[6] Mudau M, Genis A, Lochner A, Strijdom H. Endothelial dysfunction: the early predictor of atherosclerosis. Cardiovasc J Afr 2012;23(4): 222-31.

[7] Sakakura K, Nakano M, Otsuka F, Ladich E, Kolodgie FD, Virmani R. Pathophysiology of atherosclerosis plaque progression. Heart Lung Circ 2013;22(6):399-411.

[8] Shechter M, Matetzky S, Arad M, Feinberg MS, Freimark D. Vascular endothelial function predicts mortality risk in patients with advanced ischaemic chronic heart failure. Eur J Heart Fail 2009; 11(6):588-93.

[9] Hussin AM, Ashor AW, Schoenmakers I, Hill T, Mathers JC, Siervo M. Effects of vitamin D supplementation on endothelial function: a systematic review and meta-analysis of randomised clinical trials. Eur J Nutr 2017;56(3):1095-104.

[10] Borgi L, McMullan C, Wohlhueter A, Curhan GC, Fisher ND, Forman JP. Effect of vitamin D on endothelial function: a randomized, double-blind, placebo-controlled trial. Am J Hypertens 2017;30(2):124-9.

[11] Reynolds JA, Haque S, Williamson K, Ray DW, Alexander MY, Bruce IN. Vitamin D improves endothelial dysfunction and restores myeloid angiogenic cell function via reduced CXCL-10 expression in systemic lupus erythematosus. Sci Rep 2016;6:22341.

[12] Upala S, Sanguankeo A, Congrete S, Jaruvongvanich V. Effect of cholecalciferol supplementation on arterial stiffness: a systematic review and meta-analysis. Scand Cardiovasc J 2016;50(4):230-5.

[13] Rejnmark L, Bislev LS, Cashman KD, Eiríksdottir G, Gaksch M, Grübler M, et al. Non-skeletal health effects of vitamin D 
supplementation: a systematic review on findings from metaanalyses summarizing trial data. PLoS One 2017;12(7):e0180512.

[14] Breslavsky A, Frand J, Matas Z, Boaz M, Barnea Z, Shargorodsky M. Effect of high doses of vitamin D on arterial properties, adiponectin, leptin and glucose homeostasis in type 2 diabetic patients. Clin Nutr 2013;32(6):970-5.

[15] Kumar V, Yadav AK, Lal A, Kumar V, Singhal M, Billot L, et al. A randomized trial of vitamin $\mathrm{D}$ supplementation on vascular function in CKD. J Am Soc Nephrol 2017;28(10):3100-8.

[16] Raed A, Bhagatwala J, Zhu H, Pollock NK, Parikh SJ, Huang Y, et al. Dose responses of vitamin D3 supplementation on arterial stiffness in overweight African Americans with vitamin D deficiency: a placebo controlled randomized trial. PLoS One 2017;12(12): e0188424.

[17] Deluca HF, Cantorna MT. Vitamin D: its role and uses in immunology. FASEB J 2001;15(14):2579-85.

[18] Mangge H, Becker K, Fuchs D, Gostner JM. Antioxidants, inflammation and cardiovascular disease. World J Cardiol 2014; 6(6):462.

[19] Mazidi M, Karimi E, Rezaie P, Vatanparast H. The impact of vitamin D supplement intake on vascular endothelial function; a systematic review and meta-analysis of randomized controlled trials. Food Nutr Res 2017;61(1):1273574.

[20] Mirhosseini N, Rainsbury J, Kimball SM. Vitamin D supplementation, serum $25(\mathrm{OH})$ D concentrations and cardiovascular disease risk factors: a systematic review and meta-analysis. Front Cardiovasc Med 2018;5.

[21] Stojanović M, Radenković M. Vitamin D versus placebo in improvement of endothelial dysfunction: a meta-analysis of randomized clinical trials. Cardiovasc Ther 2015;33(3):145-54.

[22] Tabrizi R, Vakili S, Lankarani KB, Akbari M, Jamilian M, Mahdizadeh Z, et al. The effects of vitamin D supplementation on markers related to endothelial function among patients with metabolic syndrome and related disorders: a systematic review and meta-analysis of clinical trials. Horm Metab Res 2018;50(08): 587-96.

[23] Beveridge LA, Khan F, Struthers AD, Armitage J, Barchetta I, Bressendorff I, et al. Effect of vitamin D supplementation on markers of vascular function: a systematic review and individual participant meta-analysis. J Am Heart Assoc 2018;7(11):e008273.

[24] Higgins JP, Thompson SG, Deeks JJ, Altman DG. Measuring inconsistency in meta-analyses. BMJ: Br Med J 2003;327(7414): 557.

[25] Wan X, Wang W, Liu J, Tong T. Estimating the sample mean and standard deviation from the sample size, median, range and/or interquartile range. BMC Med Res Methodol 2014;14(1):135.

[26] Cummings P. Arguments for and against standardized mean differences (effect sizes). Arch Pediatr Adolesc Med 2011;165(7): $592-6$.

[27] Baker WL, White CM, Cappelleri JC, Kluger J, Coleman CI. Understanding heterogeneity in meta-analysis: the role of metaregression. Int J Clin Pract 2009;63(10):1426-34.

[28] Egger M, Smith GD, Schneider M, Minder C. Bias in meta-analysis detected by a simple, graphical test. BMJ 1997;315(7109):629-34.

[29] Jadad AR, Moore RA, Carroll D, Jenkinson C, Reynolds DJM, Gavaghan DJ, et al. Assessing the quality of reports of randomized clinical trials: is blinding necessary? Contr Clin Trials 1996;17(1): $1-12$.

[30] Barchetta I, Del Ben M, Angelico F, Di Martino M, Fraioli A, La Torre G, et al. No effects of oral vitamin D supplementation on non-alcoholic fatty liver disease in patients with type 2 diabetes: a randomized, double-blind, placebo-controlled trial. BMC Med 2016;14(1):92.

[31] Bressendorff I, Brandi L, Schou M, Nygaard B, Frandsen NE, Rasmussen K, et al. The effect of high dose cholecalciferol on arterial stiffness and peripheral and central blood pressure in healthy humans: a randomized controlled trial. PLoS One 2016; 11(8):e0160905.

[32] Forouhi NG, Menon R, Sharp SJ, Mannan N, Timms P, Martineau A, et al. Effects of vitamin D2 or D3 supplementation on glycaemic control and cardiometabolic risk among people at risk of type 2 diabetes: results of a randomized double-blind placebo-controlled trial. Diabetes Obes Metab 2016;18(4):392-400.

[33] Garg G, Kachhawa G, Ramot R, Khadgawat R, Tandon N, Sreenivas V, et al. Effect of vitamin D supplementation on insulin kinetics and cardiovascular risk factors in polycystic ovarian syndrome: a pilot study. Endocrine Connections 2015;4(2):108-16.

[34] Gepner AD, Ramamurthy R, Krueger DC, Korcarz CE, Binkley N, Stein JH. A prospective randomized controlled trial of the effects of vitamin D supplementation on cardiovascular disease risk. PLoS One 2012;7(5):e36617.

[35] Harris RA, Pedersen-White J, Guo D-H, Stallmann-Jorgensen IS, Keeton D, Huang Y, et al. Vitamin D3 supplementation for 16 weeks improves flow-mediated dilation in overweight AfricanAmerican adults. Am J Hypertens 2011;24(5):557-62.

[36] Larsen T, Mose FH, Bech JN, Hansen AB, Pedersen EB. Effect of cholecalciferol supplementation during winter months in patients with hypertension: a randomized, placebo-controlled trial. Am J Hypertens 2012;25(11):1215-22.

[37] Longenecker CT, Hileman CO, Carman TL, Ross AC, Seydafkan S, Brown TT, et al. Vitamin D supplementation and endothelial function in vitamin D deficient HIV-infected patients: a randomized placebo-controlled trial. Antivir Ther 2012;17(4):613.

[38] Marckmann P, Agerskov H, Thineshkumar S, Bladbjerg E-M, Sidelmann JJ, Jespersen J, et al. Randomized controlled trial of cholecalciferol supplementation in chronic kidney disease patients with hypovitaminosis D. Nephrol Dial Transplant 2012; 27(9):3523-31.

[39] Martins D, Meng Y-X, Tareen N, Artaza J, Lee JE, Farodolu C, et al. The effect of short term vitamin D supplementation on the inflammatory and oxidative mediators of arterial stiffness. Health 2014;6(12):1503.

[40] Mose FH, Vase H, Larsen T, Kancir AS, Kosierkiewic R, Jonczy B, et al. Cardiovascular effects of cholecalciferol treatment in dialysis patients-a randomized controlled trial. BMC Nephrol 2014;15(1): 50.

[41] Pilz S, Gaksch M, Kienreich K, Grübler M, Verheyen N, FahrleitnerPammer A, et al. Effects of vitamin D on blood pressure and cardiovascular risk factors: a randomized controlled trial. Hypertension 2015. Hypertensionaha. 115.05319.

[42] Ryu O-H, Chung W, Lee S, Hong K-S, Choi M-G, Yoo HJ. The effect of high-dose vitamin D supplementation on insulin resistance and arterial stiffness in patients with type 2 diabetes. Korean J Intern Med 2014;29(5):620.

[43] Sluyter JD, Camargo Jr CA, Stewart AW, Waayer D, Lawes CM, Toop L, et al. Effect of monthly, high-dose, long-term vitamin D supplementation on central blood pressure parameters: a randomized controlled trial substudy. J Am Heart Assoc 2017;6(10): e006802.

[44] Stricker H, Bianda FT, Guidicelli-Nicolosi S, Limoni C, Colucci G. Effect of a single, oral, high-dose vitamin D supplementation on endothelial function in patients with peripheral arterial disease: a randomised controlled pilot study. Eur J Vasc Endovasc Surg 2012; 44(3):307-12.

[45] Sugden J, Davies J, Witham M, Morris A, Struthers A. Vitamin D improves endothelial function in patients with Type 2 diabetes mellitus and low vitamin D levels. Diabet Med 2008;25(3):320-5.

[46] Tomson J, Hin H, Emberson J, Kurien R, Lay M, Cox J, et al. Effects of vitamin $\mathrm{D}$ on blood pressure, arterial stiffness, and cardiac function in older people after 1 year: BEST-D (Biochemical Efficacy and Safety Trial of Vitamin D). J Am Heart Assoc 2017;6(10):e005707.

[47] Witham MD, Adams F, Kabir G, Kennedy G, Belch JJ, Khan F. Effect of short-term vitamin D supplementation on markers of vascular health in South Asian women living in the UK-a randomised controlled trial. Atherosclerosis 2013;230(2):293-9.

[48] Yiu Y-F, Yiu K-H, Siu C-W, Chan Y-H, Li S-W, Wong L-Y, et al. Randomized controlled trial of vitamin D supplement on endothelial function in patients with type 2 diabetes. Atherosclerosis 2013;227(1):140-6.

[49] Witham M, Adams F, McSwiggan S, Kennedy G, Kabir G, Belch J, et al. Effect of intermittent vitamin D3 on vascular function and symptoms in chronic fatigue syndrome-a randomised controlled trial. Nutr Metab Cardiovasc Dis 2015;25(3):287-94.

[50] Levin A, Tang M, Perry T, Zalunardo N, Beaulieu M, Dubland JA, et al. Randomized controlled trial for the effect of vitamin D supplementation on vascular stiffness in CKD. Clin J Am Soc Nephrol 2017;12(9):1447-60.

[51] Dreyer G, Tucker AT, Harwood SM, Pearse RM, Raftery MJ, Yaqoob MM. Ergocalciferol and microcirculatory function in chronic kidney disease and concomitant vitamin d deficiency: an 
exploratory, double blind, randomised controlled trial. PLoS One 2014:9(7):e99461.

[52] Witham M, Dove F, Sugden J, Doney A, Struthers A. The effect of vitamin $D$ replacement on markers of vascular health in stroke patients-A randomised controlled trial. Nutr Metab Cardiovasc Dis 2012;22(10):864-70.

[53] Witham MD, Price RJ, Struthers AD, Donnan PT, Messow C-M, Ford I, et al. Cholecalciferol treatment to reduce blood pressure in older patients with isolated systolic hypertension: the VitDISH randomized controlled trial. JAMA Int Med 2013a;173(18):1672-9.

[54] Witham M, Dove F, Dryburgh M, Sugden J, Morris A, Struthers A. The effect of different doses of vitamin D3 on markers of vascular health in patients with type 2 diabetes: a randomised controlled trial. Diabetologia 2010;53(10):2112-9.

[55] Bislev LS, Rødbro LL, Bech JN, Pedersen EB, Kjaergaard AD, Ladefoged SA, et al. The effect of vitamin D3 supplementation on markers of cardiovascular health in hyperparathyroid, vitamin D insufficient women: a randomized placebo-controlled trial. Endocrine 2018;62(1):182-94.

[56] Hewitt NA, O'Connor AA, O'Shaughnessy DV, Elder GJ. Effects of cholecalciferol on functional, biochemical, vascular, and quality of life outcomes in hemodialysis patients. Clin J Am Soc Nephrol 2013;8(7):1143-9.

[57] Beveridge LA, Struthers AD, Khan F, Jorde R, Scragg R, Macdonald HM, et al. Effect of vitamin D supplementation on blood pressure: a systematic review and meta-analysis incorporating individual patient data. JAMA Int Med 2015;175(5):745-54.

[58] Zoccali C, Curatola G, Panuccio V, Tripepi R, Pizzini P, Versace M et al. Paricalcitol and endothelial function in chronic kidney disease trial. Hypertension 2014. Hypertensionaha. 114.03748.

[59] Judd SE, Tangpricha V. Vitamin D deficiency and risk for cardiovascular disease. Am J Med Sci 2009;338(1):40-4.

[60] Tripkovic L, Lambert H, Hart K, Smith CP, Bucca G, Penson S, et al. Comparison of vitamin D2 and vitamin D3 supplementation in raising serum 25-hydroxyvitamin D status: a systematic review and meta-analysis-. Am J Clin Nutr 2012;95(6):1357-64.

[61] Mozos I, Marginean O. Links between vitamin D deficiency and cardiovascular diseases. BioMed Res Int 2015;2015.

[62] Calcium IoMUCtRDRIVfVDa. Dietary reference intakes for calcium and vitamin D. Washington DC: National Academies Press (US); 2011.

[63] Jones K, Assar S, Harnpanich D, Bouillon R, Lambrechts D, Prentice A, et al. $25(\mathrm{OH})$ D2 half-life is shorter than $25(\mathrm{OH})$ D3 half-life and is influenced by DBP concentration and genotype. Clin Endocrinol Metab 2014;99(9):3373-81.

[64] Van Ballegooijen AJ, Pilz S, Tomaschitz A, Grübler MR, Verheyen N. The synergistic interplay between vitamins D and $\mathrm{K}$ for bone and cardiovascular health: a narrative review. Int J Endocrinol 2017; 2017.

[65] Dou D, Yang B, Gan H, Xie D, Lei H, Ye N. Vitamin D supplementation for the improvement of vascular function in patients with chronic kidney disease: a meta-analysis of randomized controlled trials. Int Urol Nephrol 2019;51(5):851-8.

[66] Kurnatowska I, Grzelak P, Masajtis-Zagajewska A, Kaczmarska M, Stefa nczyk L, Vermeer C, et al. Vitamin D deficiency and non-lipid biomarkers of cardiovascular risk. Arch Med Sci 2017;13(4):732.

[67] Kurnatowska IGP, Masajts-Zagajewska A, et al. Effect of vitamin K2 on progression of athersclerosis and vascular calcification in nondialyzed patients with chronic kidney disease stages 3-5. Polish Arch Int Med 2015;125:631-40.

[68] Stefan N, Vozarova B, Funahashi T, Matsuzawa Y, Weyer C, Lindsay RS, et al. Plasma adiponectin concentration is associated with skeletal muscle insulin receptor tyrosine phosphorylation, and low plasma concentration precedes a decrease in whole-body insulin sensitivity in humans. Diabetes 2002;51(6):1884-8.

[69] Ahmad S, Hewett PW, Wang P, Al-Ani B, Cudmore M, Fujisawa T, et al. Direct evidence for endothelial vascular endothelial growth factor receptor-1 function in nitric oxide-mediated angiogenesis. Circ Res 2006;99(7):715-22.

[70] Tan K, Xu A, Chow W, Lam M, Ai V, Tam S, et al. Hypoadiponectinemia is associated with impaired endotheliumdependent vasodilation. J Clin Endocrinol Metab 2004;89(2): 765-9.

[71] Ye J-J, Zhou T-B, Zhang Y-F, Wang Q, Su Y-Y, Tang J-M, et al. Levels of vitamin D receptor and CYP24A1 in patients with end-stage renal disease. Afr Health Sci 2016;16(2):462-7.

[72] Takemoto F, Shinki T, Yokoyama K, Inokami T, Hara S, Yamada A, et al. Gene expression of vitamin D hydroxylase and megalin in the remnant kidney of nephrectomized rats. Kidney Int 2003; 64(2):414-20.

[73] Michaud J, Naud J, Ouimet D, Demers C, Petit J-L, Leblond FA, et al. Reduced hepatic synthesis of calcidiol in uremia. J Am Soc Nephrol 2010. ASN. 2009080815.

[74] Kosch M, Hausberg M, Vormbrock K, Kisters K, Rahn KH, Barenbrock M. Studies on flow-mediated vasodilation and intimamedia thickness of the brachial artery in patients with primary hyperparathyroidism. Am J Hypertens 2000;13(7):759-64.

[75] Norman P, Powell J. Vitamin D, shedding light on the development of disease in peripheral arteries. Arterioscler Thromb Vasc Biol 2005;25(1):39-46.

[76] Lee JH, O'Keefe JH, Bell D, Hensrud DD, Holick MF. Vitamin D deficiency: an important, common, and easily treatable cardiovascular risk factor? J Am Coll Cardiol 2008;52(24):1949-56.

[77] Kunadian V, Ford GA, Bawamia B, Qiu W, Manson JE. Vitamin D deficiency and coronary artery disease: a review of the evidence. Am Heart J 2014;167(3):283-91.

[78] Ferder M, Inserra F, Manucha W, Ferder L. The world pandemic of vitamin D deficiency could possibly be explained by cellular inflammatory response activity induced by the renin-angiotensin system. Am J Physiol Cell Physiol 2013;304(11):C1027-39.

[79] Council NHaMR. Vitamin D nutrient reference values. 2018. 